\title{
Inverted V sign
}

\author{
Eleni M. Maroudas, ${ }^{1,3}$ Andres R. Ayoob, ${ }^{2,4}$ David J. DiSantis ${ }^{2,4}$ \\ ${ }^{1}$ University of Kentucky College of Medicine, Lexington, KY 40536, USA \\ ${ }^{2}$ Department of Radiology, University of Kentucky Medical Center, Lexington, KY 40536, USA \\ ${ }^{3} 178$ Sioux Rd, Lexington, KY 40503, USA \\ ${ }^{4} 800$ Rose Street, Room HX-315, Lexington, KY 40536, USA
}

Despite the ever-increasing utilization of CT, a supine abdominal radiograph can still be the "presenting" imaging study for patients with acute abdominal pain. Although less sensitive than upright or left lateral decubitus views, a supine frontal abdomen view thus can

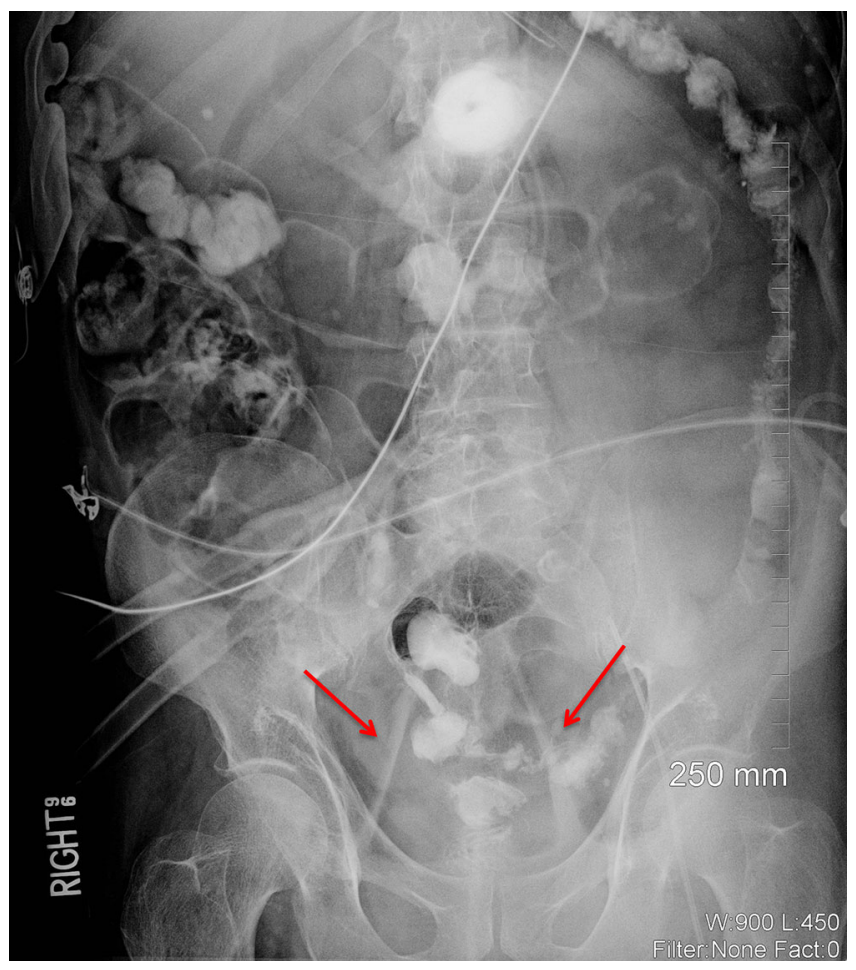

Fig. 1. "Inverted V sign." Frontal supine abdominal radiograph showing large volume pneumoperitoneum outlining pelvic peritoneal folds (arrows) (Image courtesy of Dr. Matt Maxwell). present the initial opportunity to detect pneumoperitoneum [1]. Among the many described radiographic signs is an "inverted V" visible in the pelvis (Fig. 1). Superomedially directed oblique lines are thought to represent gas outlining peritoneal folds that overlie either the obliterated umbilical arteries [2], or the inferior epigastric arteries [3]. As with any imaging evidence of pneumoperitoneum, the inverted $\mathrm{V}$ should prompt a search for perforated viscus.

Funding No funding was used for this project.

Compliance with ethical standards

Conflict of interest The authors declare that they have no conflicts of interest.

Ethical approval This article does not contain any studies with human participants or animals performed by any of the authors.

\section{References}

1. Meyers M (2000) Manifestations of intraperitoneal air. In: Meyers $\mathrm{M}$ (ed) Dynamic radiology of the abdomen normal and pathologic anatomy, 5th edn. New York: Springer

2. Weiner C, Diaconis J, Dennis J (1973) The "Inverted V": a new sign of pneumoperitoneum. Radiology 107:47-48

3. Bray F (1984) The "Inverted V" sign of pneumoperitoneum. Radiology 151:45-46 